\title{
Statements to assess diabetes knowledge (SADK) questionnaire used for Non-South-Asian patients with type 2diabetes - 'can one shoe fit all'?
}

\begin{abstract}
Education is a vital ingredient in the management of Diabetes

Background: NICE recommends well designed and structured diabetes educational programmes to all patients at the time of diagnosis and with annual updates. This article aims to discuss the importance of assessing knowledge pre and post education, as this allowed the team to ascertain existing knowledge and identified areas for development.
\end{abstract}

Aims and objectives: The Statements to Assess Diabetes Knowledge (SADK) questionnaire was used in non-South Asian patients with type 2 diabetes to assess patients' learning. It was used to identify gaps in diabetes knowledge. Learning was 'quantified' by measuring recalled information immediately post-education session and at six months during a reunion session. The tool also allowed the team, which consisted of a diabetes specialist nurse and dietician, to assess gaps in the structured education programme being delivered. The SADK was originally adapted from a Mexican tool,1 redesigned, and piloted as part of an MSc Thesis in 2005,2 to assess basic diabetes knowledge in South Asian patients with type 2 diabetes. The results of the pilot study were published in an article in 2008.3

Methods: A questionnaire Statement to Assess Diabetes Knowledge (SADK) was given to all attendees at prior to and after attending the course and again after 6months. All completed forms were collated and assessed. HbAlc results pre and post were also collected but unable to report in this article. All patients were referred into services run by Staffordshire and Stoke on Trent Partnership Trust.

Results: 203 non-South Asian patients were referred and attended for type 2 education over a 12months period from January 2012 to 2013 which was run at local sites.

i. $\quad 183(90 \%)$ of patients stated that English was their spoken or preferred language.

ii. None of the patients stated that they required an interpreter however $9 \%$ gave no response with one patient who stated Spanish.

iii. In $173(85 \%)$ of the patients the SADK scores had improved.

iv. In $14(7 \%)$ patients-had no change in their scores.

v. In 16(8\%) patients scores had declined.

vi. The biggest improvement at this stage was question 6 , 'If I am a diabetic, my children have a bigger chance of becoming diabetic'. $28 \%$ of attendees completed the questionnaire at 6 months. Of these, $81 \%$ had improved their scores at 6 months.

vii. 23 out of the 24 questions had shown further improvements at 6months compared to pre course results.

Conclusion: Findings demonstrated that the SADK questionnaire is useful in showing that learning had occurred with increased diabetes understanding. The SADK model has been shown to be an effective tool in identifying knowledge-gaps in non-South Asian patients with type 2 diabetes by assessing existing diabetes knowledge. Hence this article shows that structured education to newly diagnosed patients with diabetes has been beneficial, assessed using the SADK tool, with knowledge being absorbed and retained at 6months. Therefore it is concluded that the SADK tool is suitable for all type 2 diabetes patients to assess current knowledge and identify their educational needs; hence 'one shoe can fit all'.

Keywords: type 2 diabetes, education, SADK questionnaire, evaluation, assessment, knowledge, understanding and retaining, group education
Volume 2 Issue 5 - 2015

\author{
Angela Cross,' Harbinder Sunsoa ${ }^{2}$ \\ 'Staffordshire and Stoke on Trent Partnership, UK \\ ${ }^{2}$ Diabetes Nurse Specialist, City Hospital, UK
}

Correspondence: Harbinder Sunsoa, Diabetes Nurse Specialist, 3rd Floor, Lyng Centre, Frank Fisher Way, West Bromwich, West Midlands, B70 7AW, UK, Tel +44 0121612 2424, Fax +44 0121 612 240I, Email harbinder.sunsoa@ nhs.net

Received: October 16, 2015 | Published: December 22, 2015 


\section{Introduction}

\section{Education is the vital ingredient in the management of} diabetes ' $C$ an one shoe fit all?

Education has been and will continue to be part of the treatment for patients with diabetes. For patients with type 1 diabetes, structured programmes like Dose Adjustment for Normal Eating (DAFNE) and Bournemouth Insulin Dose Adjustment course called BERTIE have been proven to be worthwhile. ${ }^{4}$ Whilst for patients with type 2 diabetes, structured localized programmes or DESMOND/Xpert programmes have also been successful with positive evidence of leading to increase in understanding and knowledge which has promoted empowerment thus increasing in self care and management. ${ }^{4}$ Many diabetes healthcare professionals have written about the importance of patient education ${ }^{5-7}$ guidelines recommends that well-designed and structured educational programmes are cost-effective and must be offered to patients with diabetes around the time of diagnosis with annual up dates.

Education programmes often encourages dialogue between healthcare professionals and patients. Even those subdued patients, who generally sit on the back row and/or feel threatened or reluctant can indirectly benefit just by attending and listening to other patients asking questions and healthcare professionals' response, or vice verse. In fact, utilizing the SADK tool as part of the education programme has been a very positive move as it definitely encouraged patient participation. It enticed the more motivated patients who feel the need to know the answers forcing them to ask for more clarification and further explanation. For example statement 1 refers to "causes of diabetes", and in response to this statement, most patients still feel that eating too many sugary foods caused diabetes instead of knowing that it actually worsens the diabetes.

This article aims to discuss the importance of assessing knowledge pre and post education by utilizing a tool called Statements to Assess Diabetes Knowledge (SADK) in a non-South Asian population. This tool was first tested as a pilot part of an MSc thesis in 2005 and later written about the pilot study findings in an article by Sunsoa. ${ }^{3}$ This tool was initially designed for South Asian patients with type 2 diabetes and was available in audio and written formats in Urdu, Punjabi, Hindi, Gujarathi and Begali, plus in English.

The area was in South Staffordshire, predominately consisting of non-South Asian patients with type 2 diabetes. All newly diagnosed patients with diabetes are encouraged to attend the Diabetes Education Programme. To determine the level of improvement and whether the programme as had the desired effect with regard to their general diabetes control, various aspects of them controlling their condition and lifestyle were assessed. Aspects included weight management, $\mathrm{HbA1c}$ results, blood pressure and diabetes management, i.e. diet only, tablet, and insulin. Patients were also made aware of the targets for blood pressure, HbAlc and cholesterol as suggested by NICE cited in Humphries. ${ }^{8}$

\section{Aim of the project}

To enable people with Type 2 Diabetes to self management of their condition by increasing their knowledge, skills and confidence to become empowered to make more informed decisions about their lifestyle and diabetes management.

To benchmark how much newly diagnosed people with diabetes knew about the condition pre and post education course.
To determine the level of information retained a 6months post education course, in order for that the education team to assess areas of knowledge gleaned and areas that may require further development to be highlighted.

\section{Standard being measured as per the national service framework for diabetes (NICE 200I)}

A. Standard 2-The NHS will develop, implement and monitor strategies to identify people who do not know they have diabetes

B. Standard 3-Children, young people and adults with diabetes will receive a service which encourages partnership in decisionmaking, supports them in managing their diabetes and helps them to adopt and maintain a healthy lifestyle.

C. Standard 4-Adults with diabetes will receive high quality care throughout their lifetime, including support to optimise the control of their blood glucose, blood pressure and other risk factors for developing the complications of diabetes.

D. Standard 8-Young people and adults with diabetes will receive regular surveillance for the long term complications of diabetes.

\section{Methodology}

A questionnaire (Appendix A) called the Statements to Assess Diabetes Knowledge (SADK) was devised as part of another academic piece of work. ${ }^{3,9}$ The SADK questionnaire was given to all attendees of the newly diagnosed diabetes group, to be complete prior to and at the end of the course. At approximately 6months diabetes group, attendees were requested to attend a group re-union which is unique for this area. At which they were asked to complete the questionnaire for a third time, in order to gauge what diabetes education and knowledge had been retained. All of the completed questionnaires were collated and forwarded to the Trust clinical audit team for collating, monitoring and analysis of the data. The results from the 3 questionnaires are presented below.

\section{Results}

A total of 203(100\%) newly diagnosed people with type 2 diabetes attended the educational group and completed the SADK Questionnaire at the pre course and post course stage:

i. 183(90\%) stated that English was their spoken or preferred language. $18(9 \%)$ did not give a response and $1(1 \%)$ stated Spanish. No respondents stated that they required an interpreter.

ii. $173(85 \%)$ of scores had improved, $14(7 \%)$ had remained the same and $16(8 \%)$ had declined.

iii. Figure 1 above is showing the SADK score variance.

In $85 \%$ of respondents scores had shown an improvement from pre and post questionnaire results as shown in Figure 1. Regarding both the initial and post questionnaire scores, question 6 'If I am a diabetic, my children have a bigger chance of becoming diabetic', showed the greatest improvement (81) in correct answers being given as shown in Figure 1.

Note: The 2 respondents whose scores improved by 17 points were:

i. 57 year old whose score increased from 3 to 20

ii. 51 year old whose score increased from 4 to 21 


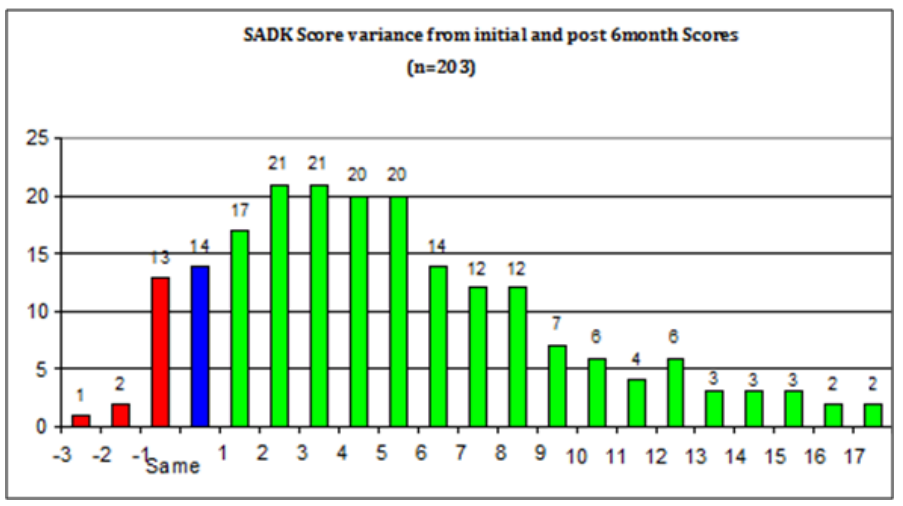

Figure I above is showing the SADK score variance.

The respondent whose score had declined by 3 points was:

a. 73 year old whose score declined from 11 to 8

In Figure $2 \mathrm{~A}$ total of $\mathrm{n}=57(28.1 \%)$ out of $\mathrm{n}=203$ course attendees completed the SADK Questionnaire at 6months post course. $\mathrm{N}=46(81 \%)$ out of $\mathrm{n}=57$ had improved from their scores on the initial questionnaire, $5(9 \%)$ had scores that remained the same, $6(10 \%)$ had scores that decreased:

Figure 2 is showing the score variance. The most predominant improvement was by $2 / 3$ points and this was evident in $42(21 \%)$ of respondents as shown in Figure 2.

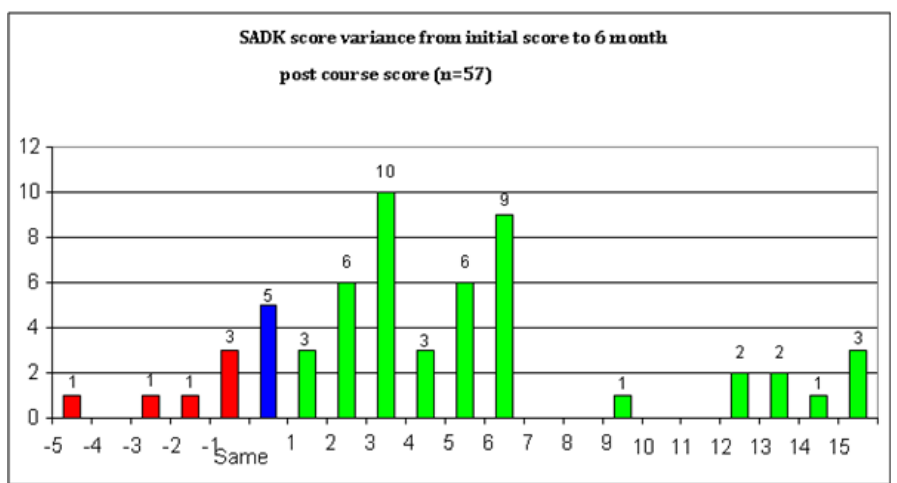

Figure 2 The score variance.

Note: The 3 respondents whose scores improved by 15 points were:
i. 68 year old whose score increased from 0 to 15
ii. 56 year old whose score increased from 5 to 20
iii. 51 year old whose score increased from 4 to 19

The respondent whose score had decreased by 5 points was

a. Age not given whose score decreased from 16 to 11

In summary every question showed an improvement in knowledge from pre to post score knowledge as shown in both Figure $1 \& 2$.

A. $46(81 \%)$ of respondents who completed the 6 month post course questionnaire had shown further improvement in their diabetes knowledge-Figure 2. This is highlighting the importance of structured education and very reassuring that $81 \%$ of the patients still retained adequate knowledge about diabetes by answering this statement correctly.
B. On the 6month post course questionnaire, Question 11 'There are 2 main types of diabetes', had a $100 \%$ correct response rateFigure 2. Very reassuring that patients' are now being able to recognise the different type of diabetes.

C. On the 6month post course questionnaire, Question 22 'Passing too much urine and feeling thirsty are signs of low blood sugars' had shown the greatest improvement (23) in correct responses - Figure 2. This is again reassuring that the symptoms being described are related to high blood glucose levels as patients have answered this statement correctly.

On the 6month post course questionnaire 23/24 questions had resulted in further improved scores from pre course results.

Only Question 15 'Cuts and injuries take time to get better in people with diabetes' did not, and had a decrease of 1, from 46 to 45 correct responses - Figure 2- this statement was included in the SADK questionnaire which was adapted from an Mexican Diabetes questionnaire $^{3}$ and it is also applicable as some of the patients may have nursed their parents or grandparents when it was a practice to clean skin before injections which is not the practice now.

\section{Conclusion}

This audit confirms that structured diabetes education provided to newly diagnosed patients with diabetes has been beneficial, and in a majority of cases new knowledge has been absorbed and retained as shown by the 6months results. This audit also has highlighted the benefits of utilising the SADK questionnaire with newly diagnosed patients with type 2 diabetes as seen in this audit results.

According to the authors, it is very important to share the results of audits with their peers, professional leads, line management, and other interested colleagues as part of sharing best practice. Furthermore, consideration was given into investigating reasons for reduction in knowledge, i.e. other medical conditions such as dementia, which may have had a bearing on results as evidenced by the results of the 73 years old patient as shown in Figure 1.

It was also apparent that utilising the results also helped the diabetes team to make informed judgements about future training and delivery of topics and aspects regarding diabetes self care and management knowledge in order to overcome gaps. Hence developed an action plan for the implementation of the recommendations accordingly and this was reflected in an agreed and shared care plan in an appropriate format and language.

Where appropriate, parents and carers should be fully engaged in this process and this was done in re-union group sessions. It is hope that the overall effects from the proposed action plan from this audit in view of NICE and NSF for diabetes this study is the evidence the improvements of patient education. This allows for modification of local patient population and specific local pathways which will increase the patient's knowledge and understanding regarding their condition, health and well being, by promoting self management and self-empowerment.

\section{Acknowledgements}

None.

\section{Conflict of interest}

Author declares that there is no conflict of interest. 


\section{References}

1. Garcia A, Villagomez A, Brown T, et al. The Starr County Diabetes Education Study. Development of the Spanish-language diabetes knowledge questionnaire. Diabetes Care. 2001;24(1):16-21.

2. Sunsoa H. Statements to Assess Diabetes Knowledge in South Asian people with type 2 diabetes. 2005 .

3. Sunsoa H. South Asian people with type 2 diabetes: a tool to assess learning. Diversity in Health and Social Care. 2008;5(1):43-53.

4. Patient. Diabetes Education and Self-management Programmes. 2015.
5. Norris S, Engelgau M, Narayan KM. Effectiveness of self-management Training in Type 2 diabetes. Diabetes Care. 2001;24(3):561-587.

6. Diabetes UK. Care Recommendation-Education of people with diabetes. 2003.

7. NICE. Type 2 diabetes-newer agents. NICE clinical guideline. 2009.

8. Humphries T. Patient education is vital for type 2 diabetes care. Guidelines in Practice. 2008.

9. Hillson R. Embarrassing diabetes. Practical Diabetes. 2014;31(8):313-314 